\title{
HIDRÓLISIS QUÍMICA Y ENZIMÁTICA DE EXTRACTO DE YACÓN (Smallanthus sonchifolius) PARA LA PRODUCCIÓN DE FRUCTOSA
}

\author{
Rosana Chirinos ${ }^{\mathrm{a}}$, Rocío Mendoza ${ }^{\mathrm{a}}$, Ana Aguilar-Gálvez ${ }^{\mathrm{a}}$, David Campos ${ }^{\mathrm{a}^{*}}$
}

\begin{abstract}
RESUMEN
Los parámetros de conversión máxima de la sacarosa y fructooligosacáridos (FOS), de un extracto clarificado de yacón ( $1 \%$ de FOS), a fructosa, fueron optimizados empleando hidrólisis química y enzimática. Se evaluó la hidrólisis química con ácido sulfúrico y ácido fosfórico; mientras que la hidrólisis enzimática fue estudiada con inulinasa e invertasa. La metodología de superficie respuesta fue empleada para la optimización de las hidrólisis. El diseño Box-Behnken fue usado para la hidrólisis química, obteniéndose como condiciones óptimas con ácido sulfúrico: $\mathrm{pH} 1$, temperatura $88,4^{\circ} \mathrm{C}$ y tiempo de $38,7 \mathrm{~min}$, alcanzando un $9,11 \mathrm{~g}$ fructosa/L de extracto; mientras que con el ácido fosfórico estas fueron: $\mathrm{pH}$ 1, temperatura $89,9^{\circ} \mathrm{C}$ y tiempo de 42,2 min obteniéndose $7,84 \mathrm{~g}$ fructosa/L de extracto. Para la hidrólisis enzimática se empleó el diseño central compuesto, encontrándose como condiciones óptimas con la inulinasa: 134 min y concentración de enzima de 11,7 U/g FOS y con la invertasa: tiempo de 127,5 min y concentración de enzima $6,74 \times 10^{-1} \mathrm{U} / \mathrm{g}$ FOS (en ambas casos se mantuvo constantes la temperatura a $50^{\circ} \mathrm{C} \mathrm{y} \mathrm{pH} 5$ ), obteniéndose un 6,16 y 7,50 g fructosa/L de extracto, respectivamente. Los modelos de superficie respuesta obtenidos mostraron un ajuste satisfactorio de los resultados para ambas hidrólisis (R2 entre $0,880$ y 0,999$)$.
\end{abstract}

Palabras clave: yacón, fructosa, hidrólisis química, hidrólisis enzimática

\section{CHEMICAL AND ENZYMATIC HYDROLYSIS OF YACON EXTRACT (Smallanthus sonchifolius) TO PRODUCE FRUCTOSE}

\begin{abstract}
The maximum conversion parameters of sucrose and fructooligosaccharides (FOS) from a clarified yacon extract ( $1 \% \mathrm{FOS})$ to fructose were optimized by using chemical and enzymatic hydrolysis. Chemical hydrolysis was performed using sulfuric and phosphoric acids and enzymatic hydrolysis with inulinase and invertase enzymes. Surface methodology response

\footnotetext{
${ }^{a}$ Instituto de Biotecnología (IBT), área de Biotecnología Industrial y Bioprocesos. Universidad Nacional Agraria La Molina (UNALM). Av. La Molina s/n, La Molina. Lima - Perú. (*) autor de correspondencia: dcampos@, lamolina.edu.pe
} 
was employed for optimization hydrolysis. A Box-Behnken design, was used for chemical hydrolysis, where optimal conditions using sulfuric acid were: $\mathrm{pH} 1$, temperature $88,4^{\circ} \mathrm{C}$ and time of 38,7 min reaching 9,11 $\mathrm{g}$ fructose/L extract while with phosphoric acids were: $\mathrm{pH} 1$, temperature $89,9^{\circ} \mathrm{C}$ and time of 42,2 min obtaining $7,84 \mathrm{~g}$ fructose/L extract. To enzymatic hydrolysis a central compose design was used, founding as optimal conditions with inulinase a time of $134 \mathrm{~min}$ and a enzymatic concentration of 11,7 U/g FOS and with invertase a time of $127,5 \mathrm{~min}$ and a enzymatic concentration of $6,74 \times 10^{-1} \mathrm{U} / \mathrm{g}$ FOS (both reactions under constant conditions of temperature $50^{\circ} \mathrm{C}$ and $\mathrm{pH}$ of 5) reaching as maximum 6,16 and 7,50 $\mathrm{g}$ fructose/L extract, respectively. The obtained response surface models produced a satisfactory fitting of the results for both hydrolysis methods ( $R 2$ entre 0,880 y 0,999$)$.

Key words: yacon, fructose, chemical hydrolysis, enzymatic hydrolysis

\section{INTRODUCCIÓN}

El yacón es una raíz originaria de los Andes, alrededor del $90 \%$ de su peso seco son carbohidratos y de estos, entre 50 al 70\% son FOS y el resto lo conforman, principalmente, sacarosa, glucosa y fructosa ${ }^{1,2}$. Los FOS son oligosacáridos de fructosa, pueden contener o no una molécula de glucosa, tienen efecto prebiótico debido a que favorecen el crecimiento de bacterias benéficas del intestino humano (bifidobacterias y lactobacilos) ${ }^{3}$.

La fructosa es comercializada en la industria alimentaria principalmente como jarabe, para ello, habitualmente, se realiza la hidrólisis enzimática del almidón hasta glucosa y posteriormente esta es isomerizada a fructosa empleando reactores con glucosa isomersa inmovilizada. Frente a este proceso, que requiere por lo menos tres etapas de hidrólisis enzimática, está la posibilidad de utilizar materias primas ricas en polisacáridos u oligosacáridos de fructosa. La hidrólisis de FOS libera cantidades importantes de fructosa ${ }^{4}$

Se han reportado diversos estudios sobre procesos de hidrólisis química y enzimática de polímeros de FOS, habiéndose considerando diversas variables: $\mathrm{pH}$, temperatura, tiempo, concentración de FOS y tipo de ácido para, la hidrólisis química ${ }^{5-7}$; así como el tipo de enzima, tiempo de hidrólisis, concentración de sustrato y concentración de enzima para la hidrólisis enzimática ${ }^{7,8}$. Los extractos de yacón, sometidos previamente a un proceso de hidrólisis, son una alternativa interesante para obtener fructosa; sin embargo a la fecha no se han encontrado estudios sobre la optimización de la hidrólisis química o enzimática de los FOS del yacón. Por esta razón, los objetivos de la presente investigación fueron: realizar un estudio comparativo de la hidrolisis química y enzimática de los FOS de yacón, teniendo en cuenta, en el primer caso, las variables $\mathrm{pH}$, tiempo y temperatura y en el segundo la concentración de enzima y tiempo de reacción. En ambos casos se utilizó la metodología de superficie respuesta (MSR). 


\section{PARTE EXPERIMENTAL}

\section{Materia prima, enzimas y reactivos}

Se empleó harina de yacón obtenida a partir de raíces de yacón, de acuerdo al procedimiento descrito por Campos et $a .^{2}$. Las enzimas empleadas fueron: la Invertase ${ }^{\circledR}((\beta$-Dfructohidrolasa- D -fructofuranosidasa) (200000 SU/g) procedente de Saccharomyces cerevisiae adquirida de NOVO Nordisk (Copenhagen, Denmark) y la inulinasa ( $\beta-\mathrm{D}-2,1-$ fructano fructohidrolasa) ( $\geq 200 \mathrm{INU} / \mathrm{g}$ ) endoinulinasa procedente de Aspergillus niger adquirida de Sigma Aldrich (USA). El ácido sulfúrico (99\%), fosfórico (85\%), ácido acético glacial, hidróxido de sodio, tartrato de sodio y potasio, hidróxido de calcio y el acetonitrilo grado HPLC fueron adquiridos de J.T. Baker (USA), el ácido 3,5 dinitrosalicílico fue adquirido de Sigma (USA).

\section{Métodos de análisis}

Determinación de sólidos solubles: Se empleó el método refractométrico 932.14-C de la $\mathrm{AOAC}^{9}$, reportándose los resultados en ${ }^{\circ}$ Brix.

Determinación del contenido de azúcares reductores: Se utilizó el método de Miller ${ }^{10}$. Los resultados se expresaron en $\mathrm{g}$ de fructosa equivalente/100 $\mathrm{mL}$ de solución.

Determinación de azúcares mediante UPLC: Los azúcares (glucosa, fructosa y sacarosa) fueron analizados de acuerdo al protocolo reportado por Campos et al. ${ }^{2}$, con ligeras modificaciones. Brevemente, los extractos de yacón (sometidos o no a hidrólisis) fueron diluidos al 50\% con acetonitrilo y posteriormente los azúcares fueron identificados y cuantificados al sistema ACQUITY UPLC H-Class (Waters, Milford, MA) equipado con un detector IR y utilizando el software Empower II. La columna empleada fue una ACQUITY UPLC ${ }^{\circledR}$ BEH Amide $(1,7 \mu \mathrm{m})$. La fase móvil estuvo compuesta por acetona: agua (77:23, $\mathrm{v} / \mathrm{v})$ con $0,05 \%$ trietilamina $(\mathrm{p} / \mathrm{v})$. Previo a la inyección al UPLC todas las muestras fueron filtradas con un filtro de $0,22 \mu \mathrm{m}$ a través de un filtro Millipore, tipo GV (Millipore, Bedford, MA). Un volumen de muestra de $2 \mu \mathrm{L}$ fue inyectado, el tiempo de corrida fue de $10 \mathrm{~min}$ a $85^{\circ} \mathrm{C}$, el flujo fue de $0,15 \mathrm{ml} / \mathrm{min}$. Los azúcares fueron identificados y cuantificados al comparar los tiempos de retención con los de los estándares previamente inyectados. La concentración de FOS fue determinada con el UPLC-IR siguiendo el procedimiento de cálculo reportado por Pedreschi et al. ${ }^{11 .}$ Los resultados se expresaron como: fructosa, glucosa, sacarosa y FOS en porcentaje.

Rendimiento de conversión de sacarosa y FOS del extracto de yacón a fructosa: Se calculó tomando en cuenta el cociente entre la cantidad de fructosa (g) liberada por litro (L) de extracto de yacón.

\section{Metodología experimental}

\section{Obtención del extracto clarificado de yacón}

La extracción se realizó con una proporción harina de yacón: agua destilada, 1:30 ( $\mathrm{g} / \mathrm{ml})$, a temperatura de $30^{\circ} \mathrm{C}$ bajo agitación constante $(150 \mathrm{rpm})$ durante $10 \mathrm{~min}$. Posteriormente, 
la mezcla fue filtrada y se recuperó el sobrenadante ${ }^{12}$. El extracto fue clarificado ${ }^{12}$, se le adicionó solución de $\mathrm{Ca}(\mathrm{OH})_{2}$ al $50 \%$ hasta llegar a $\mathrm{pH} 11$, la mezcla se calentó a $40^{\circ} \mathrm{C}$ por 5 min y luego se centrifugó a $4000 \mathrm{rpm}$ por 10 min recuperándose el sobrenadante. Este fue llevado a pH 7 con ácido fosfórico $(1 \mathrm{M})$, luego la solución fue llevada a $90^{\circ} \mathrm{C}$ por 2 minutos, seguida de centrifugación a $4000 \mathrm{rpm}$ por 10 min recuperándose el sobrenadante. El extracto clarificado fue concentrado en un evaporador rotatorio al vacío $\left(58 \pm 2{ }^{\circ} \mathrm{C}\right)$ hasta obtener la concentración deseada para luego ser empleado en las pruebas de hidrólisis.

\section{Diseño experimental y análisis estadístico}

\section{Optimización de la hidrólisis química del extracto del yacón}

La MSR fue empleada para determinar la influencia de las variables independientes: $\mathrm{pH}(1 \mathrm{a}$ 3), tiempo (20 a $70 \mathrm{~min})$ y temperatura $\left(60\right.$ a $\left.98^{\circ} \mathrm{C}\right)$. Se evaluó dos tipos de ácidos, sulfúrico y fosfórico. Las variables independientes (factores) y sus niveles $(-1,1)$ se presentan en la tabla 1. Se empleó el diseño Box Behnken (DBB), un total de 17 corridas con 5 puntos centrales fueron generadas (tabla 1). Las corridas fueron evaluadas de forma independiente para cada tipo de ácido en estudio. La respuesta medida fue la cantidad de fructosa (g) liberada a partir del extracto de yacón. Todas las corridas se realizaron por triplicado y en forma aleatoria.

\section{Optimización de la hidrólisis enzimática del extracto del yacón}

La MSR fue empleada para determinar la influencia de las variables independientes: concentración de enzima (unidades de actividad enzimática por g de FOS, U/g FOS) (de 2 a 10) y el tiempo (5 a $120 \mathrm{~min})$. Los parámetros temperatura $\left(50^{\circ} \mathrm{C}\right)$ y $\mathrm{pH}(5,0)$ fueron constantes para ambas enzimas, inulinasa e invertasa. Las variables independientes (factores) y sus niveles $(-1,1)$ se presentan en la tabla 2. Se empleó un Diseño Central Compuesto (DCC), un total de 13 corridas que incluyen 5 puntos centrales fueron generados (tabla 2). El arreglo del DCC se desarrolló independientemente para cada enzima del estudio.

En todos los ensayos se empleó un extracto de yacón con 1\% de FOS. Las superficies de respuestas generadas de ambas hidrólisis fueron experimentalmente validadas con tres repeticiones y los valores obtenidos fueron comparados con los predichos por el modelo matemático generado. Se graficó las superficies utilizando dos variables dentro del rango experimental evaluado y manteniendo la otra constante al punto central (0). El análisis estadístico se realizó con el software Statgraphics Centurion XV (Stat Point Inc., VA, USA) y Minitab® 17.1.0 (LEAD Technologies, Inc., USA). 
Tabla 1. Diseño Box Behnken para las variables independientes, niveles y variable respuesta de la hidrólisis química empleando ácido sulfúrico y ácido fosfórico

\begin{tabular}{|c|c|c|c|c|c|c|c|}
\hline \multirow{3}{*}{ Corrida } & \multirow{2}{*}{\multicolumn{3}{|c|}{ Variables independientes }} & \multicolumn{4}{|c|}{ Rendimiento, fructosa (g/L) } \\
\hline & & & & \multicolumn{2}{|c|}{$\begin{array}{l}\text { Ácido sulfúrico } \\
\text { (Y) }\end{array}$} & \multicolumn{2}{|c|}{$\begin{array}{l}\text { Ácido fosfórico } \\
\text { (Y) }\end{array}$} \\
\hline & $\begin{array}{l}\mathrm{pH} \\
\mathrm{X}_{1}\end{array}$ & $\begin{array}{c}\text { Tiempo } \\
(\min ) \\
\mathrm{X}_{2}\end{array}$ & $\begin{array}{c}\text { Temperatura } \\
\left({ }^{\circ} \mathrm{C}\right) \\
\mathrm{X}_{3}\end{array}$ & $\begin{array}{l}\text { Observado } \\
(\bar{Y})\end{array}$ & $\begin{array}{l}\text { Estimado } \\
(\hat{Y})\end{array}$ & $\begin{array}{l}\text { Observado } \\
\qquad(\bar{Y})\end{array}$ & $\begin{array}{c}\text { Estimado } \\
(\hat{Y})\end{array}$ \\
\hline 1 & $2(0)$ & $20(-1)$ & $98(1)$ & 7,84 & 6,96 & 6,24 & 5,56 \\
\hline 2 & $3(1)$ & $45(0)$ & $98(1)$ & 6,32 & 6,58 & 5,67 & 6,15 \\
\hline 3 & $2(0)$ & $45(0)$ & $79(0)$ & 6,67 & 6,81 & 5,49 & 5,86 \\
\hline 4 & $2(0)$ & $70(1)$ & $60(-1)$ & 2,06 & 2,94 & 1,69 & 2,37 \\
\hline 5 & $2(0)$ & $45(0)$ & $79(0)$ & 6,90 & 6,81 & 5,32 & 5,86 \\
\hline 6 & $1(-1)$ & $45(0)$ & $98(1)$ & 7,63 & 8,63 & 6,94 & 7,70 \\
\hline 7 & $2(0)$ & $45(0)$ & $79(0)$ & 7,34 & 6,81 & 7,36 & 5,86 \\
\hline 8 & $1(-1)$ & $45(0)$ & $60(-1)$ & 7,76 & 7,50 & 6,56 & 6,08 \\
\hline 9 & $2(0)$ & $20(-1)$ & $60(-1)$ & 0,03 & 0,40 & 0,54 & 1,09 \\
\hline 10 & $2(0)$ & $45(0)$ & $79(0)$ & 6,68 & 6,81 & 5,84 & 5,86 \\
\hline 11 & $3(1)$ & $70(1)$ & $79(0)$ & 2,27 & 2,38 & 3,32 & 3,40 \\
\hline 12 & $3(1)$ & $20(-1)$ & $79(0)$ & 0,00 & 0,62 & 0,60 & 0,81 \\
\hline 13 & $2(0)$ & $70(1)$ & $98(1)$ & 5,46 & 5,09 & 6,96 & 6,42 \\
\hline 14 & $3(1)$ & $45(0)$ & $60(-1)$ & 0,00 & 0,00 & 0,00 & 0,00 \\
\hline 15 & $1(-1)$ & $70(1)$ & $79(0)$ & 6,86 & 6,23 & 6,29 & 6,08 \\
\hline 16 & $2(0)$ & $45(0)$ & $79(0)$ & 6,46 & 6,81 & 5,30 & 5,86 \\
\hline 17 & $1(-1)$ & $20(-1)$ & $79(0)$ & 7,42 & 7,32 & 6,60 & 6,52 \\
\hline
\end{tabular}


Tabla 2. Diseño central compuesto para las variables independientes, niveles y variable respuesta de la hidrólisis enzimática empleando inulinasa e invertasa

\begin{tabular}{|c|c|c|c|c|c|c|}
\hline \multirow[b]{3}{*}{ Corridas } & \multirow{2}{*}{\multicolumn{2}{|c|}{ Variables independientes }} & \multicolumn{4}{|c|}{ Rendimiento, fructosa $(\mathrm{g} / \mathrm{L})$} \\
\hline & & & \multicolumn{2}{|c|}{$\begin{array}{c}\text { Inulinasa } \\
\text { (Y) }\end{array}$} & \multicolumn{2}{|c|}{$\begin{array}{c}\text { Invertasa } \\
(\mathbf{Y})\end{array}$} \\
\hline & $\begin{array}{c}\text { Tiempo } \\
\text { (min) } \\
\mathrm{X}_{1}\end{array}$ & $\begin{array}{c}\text { Concentración de } \\
\text { enzima (U/g } \\
\text { FOS) } \mathrm{X}_{2}\end{array}$ & $\begin{array}{l}\text { Observado } \\
(\bar{Y})\end{array}$ & $\begin{array}{c}\text { Estimado } \\
(\hat{Y})\end{array}$ & $\begin{array}{c}\text { Observado } \\
(\bar{Y})\end{array}$ & $\begin{array}{c}\text { Estimado } \\
(\hat{Y})\end{array}$ \\
\hline 1 & $85(0)$ & $0,340(-1,41)$ & 0,15 & 0,50 & 6,79 & 6,78 \\
\hline 2 & $50(-1)$ & $2(-1)$ & 1,41 & 1,10 & 6,99 & 6,99 \\
\hline 3 & $85(0)$ & $11,7(1,41)$ & 4,71 & 4,54 & 7,09 & 7,19 \\
\hline 4 & $120(1)$ & $2(-1)$ & 2,08 & 1,85 & 7,16 & 7,15 \\
\hline 5 & $50(-1)$ & $10(1)$ & 3,24 & 3,29 & 7,46 & 7,38 \\
\hline 6 & $36(-1,41)$ & $6(0)$ & 2,15 & 2,29 & 7,36 & 7,40 \\
\hline 7 & $85(0)$ & $6(0)$ & 3,41 & 3,30 & 7,67 & 7,47 \\
\hline 8 & $85(0)$ & $6(0)$ & 3,26 & 3,30 & 7,41 & 7,47 \\
\hline 9 & $120(1)$ & $10(1)$ & 5,23 & 5,36 & 7,44 & 7,35 \\
\hline 10 & $85(0)$ & $6(0)$ & 3,24 & 3,30 & 7,44 & 7,47 \\
\hline 11 & $85(0)$ & $6(0)$ & 3,36 & 3,30 & 7,41 & 7,47 \\
\hline 12 & $85(0)$ & $6(0)$ & 3,27 & 3,30 & 7,42 & 7,47 \\
\hline 13 & $134(1,41)$ & $6(0)$ & 4,23 & 4,27 & 7,44 & 7,50 \\
\hline
\end{tabular}

\section{RESULTADOS Y DISCUSIÓN}

\section{Caracterización de la harina y del extracto de yacón}

La harina de yacón empleada tuvo 34,9; 30,6 y 6,0 \% de FOS, azúcares reductores y sacarosa (en base seca, b.s.), respectivamente. Los valores fueron muy próximos a los reportados por Mejía $^{13}$ con 38,8 y $30,3 \%$ en b.s. de FOS y azúcares reductores, respectivamente. Valores mayores de $\operatorname{FOS}(55,3 \%$, b.s.) y menores de azúcares reductores $(22 \%$, b.s.) han sido reportado por Lobo et al. ${ }^{14}$ también en harina de yacón. El contenido de FOS del yacón puede variar significativamente debido a diferentes factores como: el cultivo (genotipo o cultivar), manejo del cultivo, la época de siembra y condiciones de manejo poscosecha. El extracto de yacón obtenido presentó contenidos de FOS, fructosa, glucosa y sacarosa de 24,0, 15,2, 8,3 y $4,1 \%$, respectivamente. Este extracto fue llevado a la concentración de 1\% de FOS para las pruebas de optimización de la hidrólisis química y enzimática. 


\section{Optimización de la hidrólisis química del extracto de yacón}

Los valores promedio del rendimiento de fructosa ( $\mathrm{g}$ fructosa/L extracto de yacón) observados $(\bar{Y})$ y estimados $(\hat{Y})$ para las 17 corridas generadas del DBB, para el ácido sulfúrico y fosfórico, se presentan en la tabla 1. De los resultados, se observa que para el ácido sulfúrico se obtuvo un rendimiento de fructosa entre 0 y 7,84, el mayor valor se obtuvo a pH 2, tiempo 20 min y temperatura $98^{\circ} \mathrm{C}$; mientras que para el ácido fosfórico, el rendimiento estuvo en el rango entre 0 y 6,94 ; donde el mayor valor se dio a $\mathrm{pH} 1$, tiempo 45 min y temperatura $98^{\circ} \mathrm{C}$. Los modelos matemáticos de segundo orden (cuadrático) obtenidos para este diseño fueron:

Para el ácido sulfúrico: $\mathrm{Y}=-13,0365-8,44642 \mathrm{X}_{1}+0,439742 \mathrm{X}_{2}+0,41429 \mathrm{X}_{3}-0,54485 \mathrm{X}_{1}{ }^{2}+$ $0,02843 X_{1} X_{2}+0,0849474 X_{1} X_{3}-0,00340416 X_{2}^{2}-0,00232263 X_{2} X_{3}-0,00231122 X_{3}^{2}$ (ecuación 1)

Para el ácido fosfórico: $\mathrm{Y}=-4,67908-7,51003 \mathrm{X}_{1}+0,165062 \mathrm{X}_{2}+0,2919 \mathrm{X}_{3}-0,3626 \mathrm{X}_{1}^{2}$ $+0,03026 X_{1} X_{2}+0,0696184 X_{1} X_{3}-0,00207136 X_{2}^{2}-0,000224737 X_{2} X_{3}-0,00195526 X_{3}^{2}$ (ecuación 2)

Los modelos cuadráticos fueron evaluados estadísticamente para ver el grado de ajuste a los datos experimentales. El análisis de varianza, de ambos modelos cuadráticos, indicaron que los factores: $\mathrm{pH}\left(\mathrm{X}_{1}\right)$ y temperatura $\left(\mathrm{X}_{3}\right)$, fueron significativos $(p<0,05)$, mientras que el tiempo $\left(\mathrm{X}_{2}\right)$ no resultó significativo. La suma de cuadrados del error puro resultó pequeña con respecto a la suma de cuadrados del total, indicando ello una buena reproducibilidad en el punto central ${ }^{15}$. Finalmente, respecto al coeficiente de determinación $\left(\mathrm{R}^{2}\right)$, se pudo establecer que el 96,3 y el 93,8\% de la variabilidad de los resultados, para la optimización con ácido sulfúrico y fosfórico, respectivamente, es explicado por el modelo cuadrático obtenido. Además, existió una buena correlación entre los valores experimentales y estimados $\left(\mathrm{R}^{2}=\right.$ $0,96 \mathrm{y} \mathrm{el} \mathrm{R}_{\text {ajustado }}^{2}=0,91, \mathrm{y} \mathrm{R}^{2}=0,96 \mathrm{y} \mathrm{R}_{\text {ajustado }}=0,86$, para el ácido sulfúrico y fosfórico, respectivamente).

Las figuras 1 y 2 muestran las relaciones entre los diferentes factores para las hidrólisis con ácido sulfúrico y fosfórico, respectivamente. En ambos casos se observa, que en la relación del $\mathrm{pH}$ con el tiempo (figuras 1a y $2 \mathrm{a}$ ) y el $\mathrm{pH}$ y la temperatura (figuras $1 \mathrm{~b}$ y $2 \mathrm{~b}$ ), el pH presenta un efecto con tendencia lineal de pendiente negativa, de donde se observa que el mayor rendimiento de fructosa se obtiene a valores bajos de $\mathrm{pH}(\sim \mathrm{pH} 1)$, mientras que el tiempo y la temperatura presentaron efectos cuadráticos, lo que se evidencia cuando se relacionaron las variables tiempo y temperatura (figuras 1c y 2c), encontrándose los óptimos hacia los valores medios de los rangos evaluados para la temperatura y tiempo. En las figuras $1 \mathrm{~b}$ y $2 \mathrm{~b}$ y, 1c y 2c; se puede observar que la temperatura presenta un comportamiento cuadrático con tendencia a obtener mayores valores de la variable respuesta entre 85 a $95^{\circ} \mathrm{C}$. Por otro lado, con respecto al tiempo se puede observar en las figuras 1a y $2 \mathrm{a}$ y, 1c y $2 \mathrm{c}$ que este factor muestra un comportamiento cuadrático obteniendo mejores respuestas entre 40 a 55 min. 
De acuerdo a los resultados obtenidos las condiciones óptimas, utilizando ácido sulfúrico, correspondieron a $\mathrm{pH} 1,38,7 \mathrm{~min}$ y $88,4^{\circ} \mathrm{C}$, obteniéndose un rendimiento en fructosa de 9,11 $\mathrm{g} / \mathrm{L}$ de extracto de yacón; mientras que con los ensayos de validación del modelo se obtuvo 9,00 g/L, cercano al valor estimado. De otro lado, las condiciones óptimas con el ácido fosfórico fueron $\mathrm{pH}$ de 1 , tiempo de $42,2 \mathrm{~min}$ y temperatura de $89,9^{\circ} \mathrm{C}$, obteniéndose una conversión de 7,84 g/L y la validación dio un valor experimental bastante cercano (7,53 g/L).

Los mejores resultados se obtuvieron con ácido sulfúrico. Al respecto, Sarchami y Rehmann ${ }^{7}$ obtuvieron 98,5\% de hidrólisis de la inulina usando ácido sulfúrico a $\mathrm{pH}$ 2, durante 35 min y a $97^{\circ} \mathrm{C}$; mientras que con el ácido fosfórico obtuvieron $94 \%$ de hidrólisis a $\mathrm{pH} 2$, durante 39 min y a $95^{\circ} \mathrm{C}$, atribuyendo esta diferencia a que el ácido fosfórico tiende a producir mayor cantidad furfurales a bajos valores de $\mathrm{pH}$ y altas temperaturas, en comparación con el sulfúrico y clorhídrico. Esto afecta el rendimiento en fructosa. También Szambelan y Nowak encontraron que con ácido sulfúrico se libera de 6 a 13\% más de azúcares reductores que con ácido fosfórico.

(a) Temperatura $=79^{\circ} \mathrm{C}$

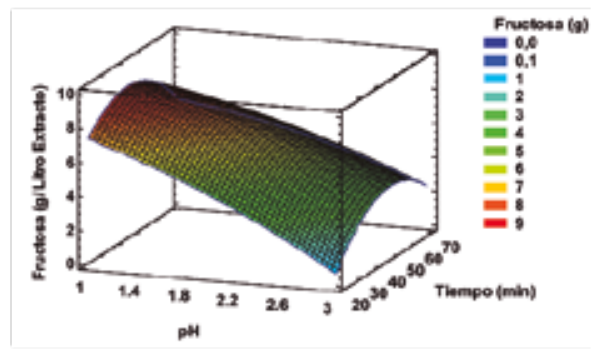

(b) Tiempo $=45 \mathrm{~min}$

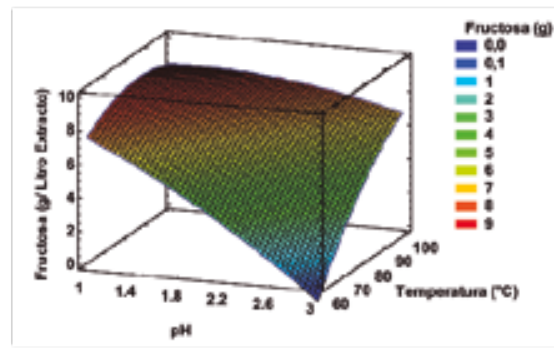

(c) $\mathrm{pH} 2,0$

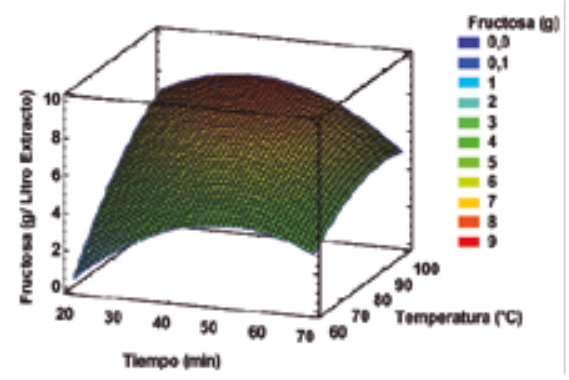

Figura 1. Gráfica de la superficie de respuesta del rendimiento de fructosa obtenido con ácido sulfúrico, para los efectos: (a) pH-tiempo, (b) $\mathrm{pH}$-temperatura y (c) tiempo-temperatura 


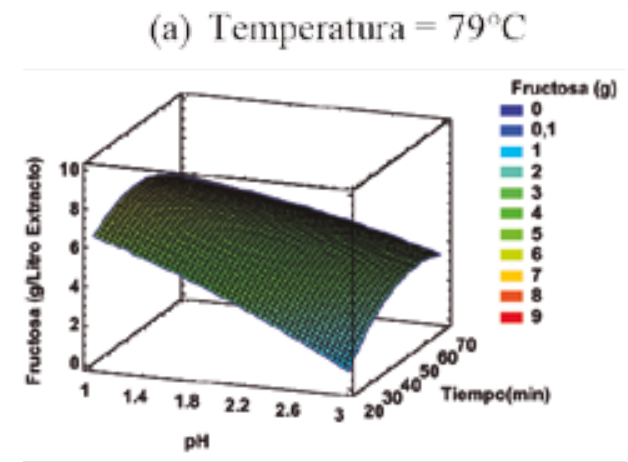

(b) Tiempo $=45 \mathrm{~min}$

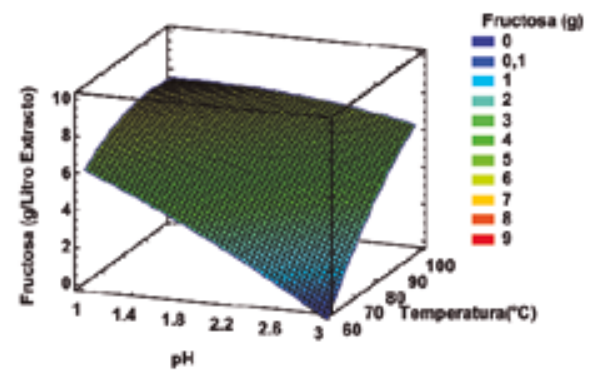

(c) $\mathrm{pH}=2,0$

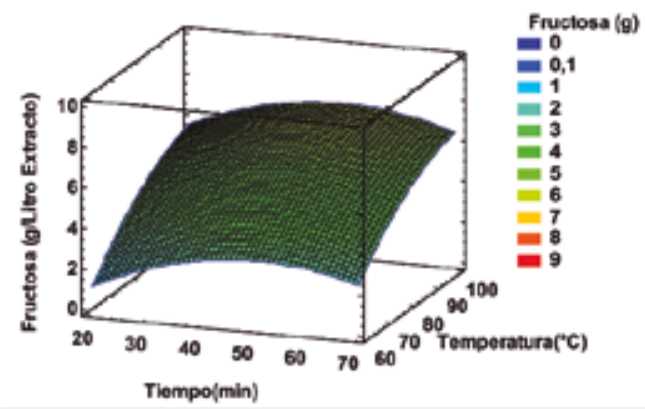

Figura 2. Gráfica de la superficie de respuesta del rendimiento de fructosa obtenido con ácido fosfórico, para los efectos: (a) pH-tiempo, (b) pH-temperatura y (c) tiempo-temperatura

De otro lado, es conocido que los azúcares se pueden degradar cuando son sometidos a condiciones drásticas de temperatura y $\mathrm{pH}$, produciendo compuestos tóxicos como furfurales 5 ; siendo por tanto una alternativa la hidrolisis enzimática.

\section{Optimización de la hidrólisis enzimática del extracto de yacón}

Los resultados promedio del rendimiento en fructosa ( $\mathrm{g} / \mathrm{L}$ de extracto de yacón) observados $(\hat{Y})$ y estimados $(Y)$ obtenidos con inulinasa e invertasa, se presentan en la tabla 2. Se observa que los valores obtenidos con inulinasa e invertasa estuvieron entre 0,15 - 5,23 y 6,79 - 7,67 $\mathrm{g} / \mathrm{L}$, respectivamente; los mayores valores se obtuvieron bajo las siguientes condiciones: 10 $\mathrm{U}$ inulinasa/g FOS por $120 \mathrm{~min}$ y de $6 \times 10^{-1} \mathrm{U}$ invertasa/g FOS por $85 \mathrm{~min}$.

Los modelos matemáticos de segundo orden obtenidos para este diseño fueron:

Para la inulinasa: $Y=-0,295841+0,00765898 X_{1}+0,450906 X_{2}-0,00000979331 X_{1}^{2}+$ $0,00235536 \mathrm{X}_{1} \mathrm{X}_{2}-0,0245293 \mathrm{X}_{2}^{2}$

Para la invertasa: $Y=6,39784^{2}+0,00432489 X_{1}+2,47059 X_{2}-0,0000077251 X_{1}^{2}-$ $0,00345179 \mathrm{X}_{1} \mathrm{X}_{2}-1,50936 \mathrm{X}_{2}^{2}$

(ecuación 4) 
El modelo cuadrático, derivado del diseño DCC, fue evaluado estadísticamente para ver el grado de ajuste con los datos experimentales. El análisis de varianza de los modelos cuadráticos (ecuaciones 3 y 4), indican que los factores tiempo y concentración de enzima fueron significativos para la inulinasa; mientras que para la invertasa, solo la concentración de enzima fue significativo. También en función a los valores obtenidos de la suma de cuadrados del error puro y la suma de cuadrados del total, se presentó una buena reproducibilidad en el punto central. El coeficiente de determinación $\left(\mathrm{R}^{2}\right)$, para las enzimas inulinasa e invertasa, indican que el $98,3 \%$ y el $88,0 \%$ de la variabilidad de los resultados, es explicado por el modelo cuadrático respectivo; mientras que altos grados de correlación, entre los valores experimentales y estimados, también fueron encontrados $\left(\mathrm{R}^{2}=0,98 \mathrm{y} \mathrm{R}^{2}\right.$ ajustado $=0,97, \mathrm{y} \mathrm{R}^{2}=$ $\left.0,88 \mathrm{y} \mathrm{el} \mathrm{R}_{\text {ajustado }}^{2}=0,80\right)$, para la inulinasa e invertasa, respectivamente.

Las figuras $3 \mathrm{a}$ y $3 \mathrm{~b}$ muestran las relaciones obtenidas entre los factores tiempo y concentración de enzima para inulinasa e invertasa, respectivamente. Se observa que, en ambos casos, el factor tiempo tiene un efecto lineal con pendiente positiva, obteniéndose los mayores valores de rendimiento en fructosa a los mayores tiempos de hidrólisis, mientras que para el caso de la concentración de enzima se observa un efecto curvatura bastante marcado cuando se emplea invertasa, respecto al empleo de la inulinasa. Las condiciones óptimas de hidrólisis con inulinasa e invertasa se obtuvieron con 11,7 U/g FOS y $134 \mathrm{~min}$ con un rendimiento en fructosa de $6,16 \mathrm{~g} / \mathrm{L}$ de extracto $\mathrm{y}$, de $6,74 \mathrm{x}$ 10-1 U/g FOS y 127,5 min, con un rendimiento de 7,50 g/L de extracto, respectivamente. Se encontró que los valores experimentales $(6,14 \mathrm{y}$ 7,46 g/L de extracto, para inulinasa e invertasa, respectivamente) obtenidos son muy cercanos a los valores estimados.

a)

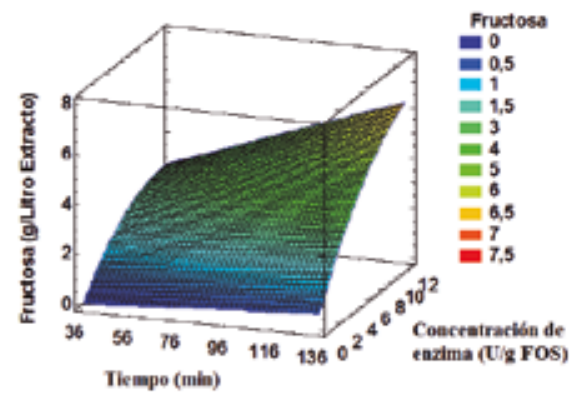

b)

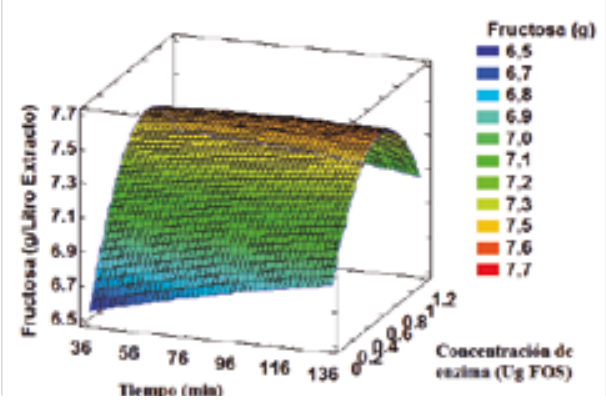

Figura 3. Gráfica de la superficie de respuesta para los efectos tiempo - concentración de enzima en el rendimiento de fructosa con inulinasa (a) e invertasa (b)

Los resultados muestran que con la invertasa se obtiene un mayor rendimiento en fructosa. Debemos indicar que, la inulinasa utilizada es del tipo endoenzima, cuya característica principal es hidrolizar los enlaces glucosídicos al interior de la molécula. En diversos estudios se ha observado que este tipo de enzima es inhibida por los productos de la reacción, dando una baja actividad cuando en el medio se encuentran FOS de bajo grado de polimerización ${ }^{16,17}$. Por lo tanto, resulta evidente que es necesario mayor cantidad de inulinasa para hidrolizar los 
FOS de yacón que son de bajo grado de polimerización. Montañez et al. ${ }^{16} \mathrm{y}$ Li et al. ${ }^{18}$, han demostrado que la invertasa proveniente de $S$. cerevisiae es una enzima capaz de hidrolizar los enlaces $\beta(1 \rightarrow 2)$ de los FOS, aun siendo estos de bajo grado de polimerización. Por otra parte, con respecto a la inulinasa del tipo endo, Montañez et al. ${ }^{16}$ y Liu et al. ${ }^{17}$ afirman que esta enzima hidroliza mejor los FOS de mayor grado de polimerización y que es inhibida por los FOS de grado de polimerización. Por lo tanto, de lo mencionado, se deduce que las actividades de ambas enzimas están influenciadas por el grado de polimerización de los FOS. Szambelan y Nowak ${ }^{8}$ compararon la actividad de la inulinasa e invertasa para la hidrólisis de la inulina, en donde se observó que el rendimiento en fructosa usando invertasa es 45 por ciento menor al valor obtenido usando inulinasa comercial. Sin embargo, en este estudio, al utilizar invertasa, se obtiene $1,34 \mathrm{~g}$ de fructosa/L de extracto más que con la inulinasa. La diferencia entre ambos comportamientos se debe al grado de polimerización del sustrato.

\section{Rendimiento en fructosa}

En la tabla 3 se presenta los rendimientos obtenidos bajo las condiciones óptimas de hidrólisis expresados en fructosa liberada a partir de la FOS, la sacarosa y fructosa total (liberada + inicial). De la tabla se observa que el más alto rendimiento alcanzado correspondió a la hidrólisis con ácido sulfúrico; mientras que para la hidrólisis enzimática el mayor rendimiento se obtuvo con invertasa. Es importante recalcar que las condiciones drásticas a las que están sometidos los azúcares en la hidrólisis química producen la deshidratación de estos, formando furfurales ${ }^{5,6}$ así como otros productos químicos derivados, siendo por ello recomendable realizar la hidrólisis enzimática.

Tabla 3. Rendimiento en fructosa de las hidrólisis química y enzimática realizadas bajo las condiciones óptimas en extracto de yacón

\begin{tabular}{lll}
\hline \multicolumn{1}{c}{$\begin{array}{c}\text { Condiciones óptimas de } \\
\text { hidrólisis }\end{array}$} & $\begin{array}{c}\text { Rendimiento en fructosa } \\
\text { (fructosa liberada) } \\
(\mathrm{g} / \mathrm{L} \text { de extracto })\end{array}$ & $\begin{array}{c}\text { Rendimiento en fructosa } \\
\text { (fructosa total)* }\end{array}$ \\
& 9,11 & 15,52 \\
\hline Ácido sulfúrico: $\mathrm{pH} 1,0 ;$ & & 14,24 \\
88,4 min; $38,7^{\circ} \mathrm{C}$ & 7,84 & 12,56 \\
Ácido fosfórico: $\mathrm{pH} 1,0 ;$ & \\
89,9 min; $42,2^{\circ} \mathrm{C}$ & \\
Inulinasa: $134 \mathrm{~min} ; 11,7 \mathrm{U} / \mathrm{g}$ & 6,16 & 13,91 \\
FOS & 7,50 & \\
Invertasa: 127,$5 ; 6.74 \times 10^{-1}$ & & \\
U/g FOS
\end{tabular}

Representa la suma de fructosa inicial y fructosa liberada con la hidrólisis 


\section{CONCLUSIONES}

El proceso de optimización de superficie respuesta para la hidrólisis química, utilizando el diseño Box Behnken, indicó que los factores $\mathrm{pH}$ y temperatura influyeron significativamente en el rendimiento en fructosa a partir de un extracto de yacón con $1 \%$ de FOS, mientras que el factor tiempo no fue significativo; adicionalmente el tipo de ácido también influye en el rendimiento en fructosa obtenida, así, bajo las condiciones óptimas encontradas por los modelos cuadráticos, el ácido sulfúrico produjo un mayor rendimiento en fructosa $(9,11 \mathrm{~g} / \mathrm{L}$ de extracto de yacón) en comparación al ácido fosfórico $(7,84 \mathrm{~g}$ fructosa/ L de extracto de yacón).

El proceso de optimización de superficie respuesta para la hidrólisis enzimática, utilizando el diseño central compuesto, indicó que el factor, concentración de enzima, influyó significativamente en el rendimiento en fructosa; mientras que factor tiempo resultó significativo para inulinasa pero no para invertasa. Con invertasa se obtuvo mayor rendimiento en fructosa $(7,50 \mathrm{~g}$ fructosa/L de extracto de yacón) en comparación a la inulinasa $(6,16 \mathrm{~g}$ fructosa/ L de extracto de yacón).

\section{AGRADECIMIENTO}

Al Programa Nacional de Innovación para la Competitividad y Productividad (Innóvate Perú), por el financiamiento para el desarrollo de la presente investigación como parte del proyecto "Optimización del proceso de extracción de los fructooligosacáridos de yacón (Smallanthus sonchifolius) y de su purificación mediante técnicas cromatográficas y de biotransformación enzimática" (Contrato $N^{\circ}$ 142-FINCyT-IA-2013)

\section{BIBLIOGRAFÍA}

1. Hermann M, Freire I, Pazos C. Compositional diversity of the yacon storage root. Lima: Centro Internacional de la Papa (CIP); 1999.

2. Campos D, Aguilar A, Pedreschi R. Stability of fructooligosaccharides, sugars and color of yacon (Smallanthus sonchifolius) roots during blanching and drying. Int J Food Sci Tech. 2016; 51: 1177-1185.

3. Vaňková K, Polakovič M. Optimization of single-colum chromatographic separation of fructooligosaccharides. Process Biochem. 2010; 45: 1325-1329.

4. Seminario J, Valderrama M, Manrique I. El yacón: Fundamentos para el aprovechamiento de un recurso promisorio. Lima: Centro Internacional de la Papa (CIP), Universidad de Cajamarca, Agencia Suiza para el Desarrollo y la Cooperación (COSUDE); 2003.

5. Pekic B, Slavica Z, Lepojevic Z, Petrovic S. Effect of the Acid Hydrolysis of Jerusalem Artichoke Inulin. Food Chem. 1984; 17: 169-173.

6. Blecker C, Fougnies C, Van Herck J, Chevalier J, Paquot M. Kinetic Study of the acid hydrolysis of various oligofructose samples. J Agric Food Chem. 2002; 50: 1602-1607. 
7. Sarchami T, Rehmann L. Optimizing enzymatic hydrolysis of inulin from Jerusalem artichoke tubers for fermentative butanol production. Biomass and Bioenergy. 2014; 69: 175- 182.

8. Szambelan K, Nowak J. Acid and enzymatic hydrolysis of Jerusalem Artichoke (Helianthus tuberosus L.) tubers for further etanol production. EJPAU. 2006; 9: 36.

9. AOAC (Association of Official Analytical Chemists). Official Methods of Analysis. Washington D.C.: Association of Official Analytical Chemists;1995.

10. Miller G. Use of dinitrosalicylic acid reagent for determination of reducing sugar. Anal Chem. 1959; 31: 426-428.

11. Pedreschi R, Campos D, Noratto G, Chirinos R, Cisneros-Zevallos L. Andean Yacon Roots (Smallanthus sonchifolius Poepp. Endl) Fructooligosaccharides as a Potential Novel Source of Prebiotics. J Agric Food Chem. 2003; 51: 5278- 5284.

12. Inga M, Betalleluz I, Kina M, Campos D. Optimización del proceso de extracción de los fructooligosacaridos de yacón (Smallantus sonchifolius). Rev Soc Quím Perú. 2015; 81: 263-272.

13. Mejía R. Impregnación al vacío de fructooligosacáridos de yacón (Smallanthus sonchifolius Poepp \& Endl.) en manzana. [Tesis Ingeniero en Industrias Alimentarias]. Lima: Universidad Nacional Agraria La Molina; 2015.

14. Lobo A, Colli C, Alvares E, Filisetti T. Effects of fructans-containing yacon (Smallanthus sonchifolius Poepp \& Endl.) flour on caecum mucosal morphometry, calcium and magnesium balance, and bone calcium retention in growing rats. Br J Nutr. 2007; 97: 776-785.

15. Ayala J, Pardo R. Optimización por diseños experimentales con aplicaciones en ingeniería. Lima: CONCYTEC; 1995.

16. Montañez S JL, Venegas GJ, Bernardino NA, Ramos REG. Enzymatic production of high frcutose syrup from Agave tequilana fructans and its physicochemical characterization. African J Biotech. 2011; 10: 19137- 19143.

17. Liu Y, Zhou SH, Cheng YR, Chi Z, Chi ZM, Liu GL. Synergistic effect between the recombinant exo- inulinase and endo- inulinase on inulin hydrolysis. J Mol Catal B: Enzym. 2016; 120: 27- 38.

18. Li Y, Fu WJ, Liu NN, Tan MJ, Liu GL, Chi ZM. Role of SUC2 gene and invertase of Saccharomyces sp. W0 in inulin hydrolysis. J Mol Catal B: Enzym. 2015; 111: 71- 78. 